(C) Elsevier Sequoia S.A., Lausanne - Printed in the Netherlands

\title{
PREDICTABILITY OF PROTOTYPE MACHINE LIQUID CAVITATION AND DROPLET IMPACT EROSION FROM LABORATORY TESTS*
}

\author{
F. G. HAMMITT, J.-B. HWANG, S. A. BARBER and M. K. DE \\ Cavitation and Multiphase Flow Laboratory, Mechanical Engineering Department, \\ University of Michigan, Ann Arbor, Michigan (U.S.A.)
}

(Received July 5, 1977)

\section{Summary}

Computer models of cavitation bubble collapse and also of liquid jet and droplet impact are described. These can predict local stresses and strains as a function of time in the impacted material, but not as yet the erosion rates to be anticipated. A pressure pulse spectrum measuring technique for a priori prediction of eventual erosion rates is described. Initial results, which appear very hopeful, are described.

\section{Introduction}

One of the major problems at present concerning liquid cavitation and impact erosion is our relative inability to predict erosion rates, or even the likely existence of important erosion, in field devices from available laboratory tests or theoretical models. This problem exists for all liquids of interest ranging from water to liquid metals such as sodium required as coolant for nuclear fast breeder reactors. Even the relative erosion resistance of materials measured in the same type of facility but with minor differences in operating parameters are often not the same even to a useful engineering approximation. In addition, there is no presently known technique for applying this type of test result to field devices. This was illustrated forcefully in a recent round-robin conducted by ASTM Committee G-2 [1] for the simplest and most readily standardizable type of cavitation test device [2].

A similar situation exists for liquid impact erosion, for which a second ASTM round-robin has been held [3]. The liquid impact situation is even more difficult to standardize because of the present lack of a relatively conventional test device, a situation which is even worse for the case of solid particle impact (which is not within the scope of the present paper).

*Paper presented at the International Conference on the Wear of Materials, St. Louis, Mo., U.S.A. $26-28$ April, 1977. 
This paper attempts to summarize the status of liquid erosion laboratory testing and its relation to erosion in field machines. In addition, some of the pertinent experimental and theoretical work being done at the University of Michigan to improve this situation is summarized.

One of the major difficulties involved in the prediction of field machine erosion from laboratory tests is that liquid erosion involves both mechanical and corrosive effects. In long term field exposure the corrosion effects can be relatively much more important than in the laboratory devices which in general are intended to accelerate the mechanical effects and thus to provide tests of short enough duration to be feasible from the viewpoint of laboratory tests. To provide realistic modelling for specific field applications it is necessary either to accelerate both modes of attack in suitable proportion or to provide methods of measuring them separately, i.e. to identify somehow the mechanical and corrosive effects in laboratory tests so that a more meaningful application to field devices can be made. Our work to achieve these goals will be described further in the paper.

One objective then is to measure, with greater precision than is now possible, the characteristics of the cavitation or liquid impact regime in both laboratory and field devices and thus to make more meaningful predictions than are now possible of probable prototype field results from laboratory tests. To accomplish this goal we are attempting to measure and count actual individual pressure pulses from bubble collapse originating from the cavitation field in both laboratory and field devices. This will have the advantage of increasing basic understanding of the cavitation fields involved and also of providing a comparison of their "intensity" in various field and laboratory devices.

Liquid impingement is now supposed $[2,4]$ to be a major contributory mechanism in cavitation erosion through the asymmetric collapse of bubbles and the generation of a liquid microjet which impinges upon the material surface. We have therefore developed various numerical models of individual droplet or jet impact, with the goal of providing the capability for estimating actual stresses and strains in a material so impacted. These models of course apply to direct droplet or jet impact as well as to cavitation. Hopefully, it may eventually become possible to compute expected rates of erosion if the size, velocity, shape and number of impacting droplets is known. The likelihood of a realistic calculating capability of this type for droplet impact appears to be much greater than for cavitation erosion because in most cases the details of the attacking flow regime, i.e. microjet, pressure pulses etc., are known much more precisely. It is for this reason that our present efforts on cavitation are primarily concerned with providing a technique for measuring and counting bubble collapse pulses. These spectra can hopefully be used as a measure of the "intensity" of the cavitation regime attack. Some details of our mathematical modelling of liquid impact will also be provided in the paper. 


\section{Droplet and jet impact - computer modelling}

\section{General background}

The phenomenon of liquid-solid impact has technological importance in various engineering applications including steam turbines, rain erosion of aircraft or missile components and also cavitation through the liquid microjet mechanism previously discussed. Its detailed study using sophisticated computer models is thus worth while in attempting to promote the ability to predict damage. It has thus been pursued vigorously in this laboratory over the past several years [5 - 11]. Previously we had also conducted several studies of bubble collapse utilizing both high speed photography [12 - 14] and computer models [15 - 19]. Some of these studies [12, 13, 19] considered spherically symmetric bubble collapse, but included all pertinent real-fluid effects, including thermodynamic restraints [19]. Our other studies considered the effects of non-symmetrical influences upon bubble collapse [14-18], such as the presence of adjacent walls, pressure gradients, relative (slip) velocity around the collapsing bubble and multibubble effects $[16,17]$, but neglected real-fluid effects other than viscosity. Photographic studies here [14-16] and elsewhere [20,21] had shown the controlling importance of these non-symmetric effects in many cases. Unfortunately it does not appear feasible at this point to include asymmetry and real-fluid effects or the effects of multiple bubbles together in a completely general computer program.

In summary [22] the results of the above and other recent studies indicate the probable predominance of the liquid microjet mechanism in cavitation damage. It is possible to estimate from these studies the probable velocity (about $300 \mathrm{~m} \mathrm{~s}^{-1}$ ) and diameter (about $1-10 \mu \mathrm{m}$ ) of such microjets in typical cases. Hence the microjet velocity magnitude is roughly typical of other droplet or jet impact applications, such as the steam turbine droplet erosion problem, but its diameter is orders of magnitude less since droplet diameters of the order of $1 \mathrm{~mm}$ are typical in conventional droplet erosion problems. Hence it may be assumed that cavitation and conventional droplet erosion are very similar in mechanism as well as appearance, although the cavitation erosion may be generally of finer texture, and this has in fact been the general observation for many years.

\section{Liquid impact computer studies \\ General}

In recent years, as previously indicated, our primary effort concerning computer modelling studies has been concentrated upon the modelling of droplet and jet impact. While this work has been reported in detail elsewhere [5 - 10] it is useful to present a brief review here of the situation.

To a first approximation, the liquid-solid impact process, as it applies to material erosion, is essentially a non-steady process (fundamentally that classically termed water hammer) and governed by*

\footnotetext{
*The zero subscripts indicate ambient liquid properties.
} 


$$
\Delta P_{\mathrm{WH}}=\rho_{0} C_{0} V / g
$$

where $\Delta P_{\mathrm{wH}}$ is the resultant pressure rise, $\rho$ the liquid density, $C$ the pressure wave velocity (approximately equal to the sonic velocity) in the liquid and $V$ the perpendicular component of impact velocity.

This equation would be almost exactly correct for an impact between two semi-infinite planes of liquid and solid. However, for drops or jets of finite size and/or curved leading surfaces, there will obviously be corrections related to droplet shape. In addition there are important corrections due to changing liquid properties, such as $\rho$ and $C$, under the high pressures induced by the collision itself as well as corrections due to the non-rigidity of the target material.

If the process were essentially steady state, as would be the case for the impact of an elongated jet (many $L / D$ ratios), the pressure along the impacted surface would very quickly fall from water-hammer pressure magnitude to that of stagnation pressure

$$
\Delta P_{\mathrm{st}}=\rho_{0} V^{2} / 2
$$

which is in general much smaller, but is the highest pressure attainable in a steady process.

\section{Geometrical effects}

The perpendicular collision between flat semi-infinite liquid and solid planes is entirely analogous to the sudden deceleration of a liquid column in a rigid pipe, and hence the classical water-hammer equation describes the phenomenon correctly. However, the collision of finite flat or curved surfaces involves important differences. For a finite flat liquid surface the effect of angle of impact is much more difficult to analyze than the others, since the phenomenon is no longer axially symmetric. Nevertheless, all of these situations have been investigated to some extent in our computer studies [5 - 11].

The situations investigated here for rigid flat surfaces include:

(a) spherical and combined flat and spherical shapes (Fig. 1);

(b) right-circular cylinder impacting in a direction parallel to axis;

(c) conical droplet impacting in a direction parallel to axis. This allows the investigation of a constant contact angle of impact, as opposed to the situation with an impacting spherical drop, where the contact impact angle increases from zero to $90^{\circ}$ as the collision proceeds.

The spherical and combined flat-spherical shapes (Fig. 1) were investigated, since these shapes match most closely many actual droplet shapes in such important impingement cases as aircraft rain erosion and turbine wet steam erosion. Impact with the leading edge of a right-circular cylindrical drop was included to model as closely as feasible impact with elongated jets such as the liquid microjet originating from cavitation bubble collapse, as well as to provide a simple limiting case where acute contact angle effects, which are present with spherical drops, are absent. Finally, the conical drop was investigated as another relatively simple (axially symmetric) 


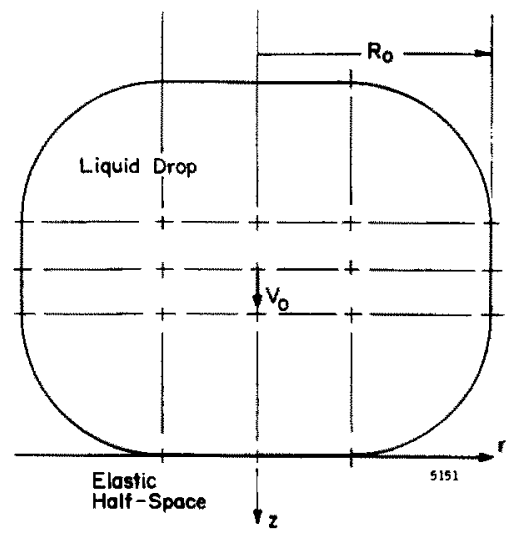

Fig. 1. Problem geometry.

limiting case where an acute angle effect is present, but the angle is constant. This allows comparison with previous more simplified analyses which still take into account the contact angle effect [23].

In general, the effect of the contact angle, as for a spherical drop for example, is to provide a non-uniform pressure distribution along the impact surface which reaches a maximum away from the axis and near the center of contact, i.e. at increasing radius as the collision proceeds. While the waterhammer model (eqn. (1)) still provides a good approximation of the average pressure along the contact surface at any instant during the impact, the point pressure increases toward the outside of the contact region and is a minimum along the axis (Fig. 2). According to our model, it exceeds the classical water-hammer pressure only slightly. Some experimental information (Fig. 3) indicates that our calculation may be somewhat conservative in this regard and that the actual peak pressure may be up to 1.5 times the water-

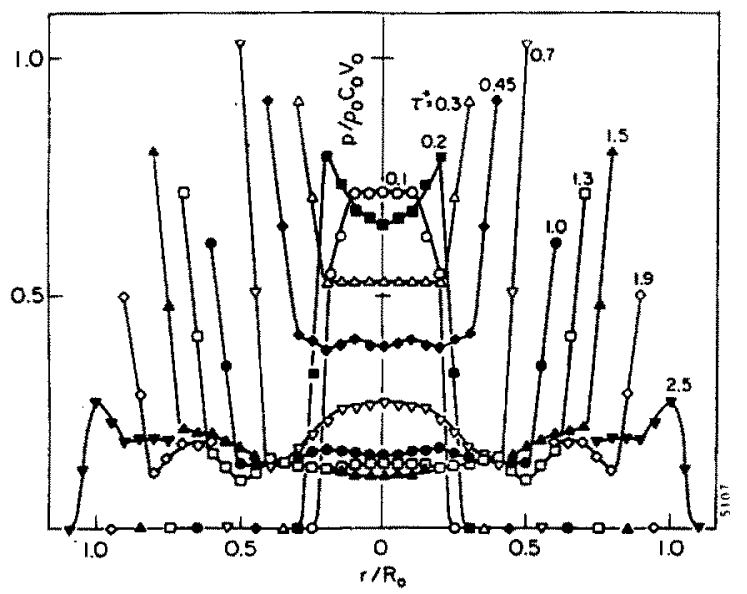

Fig. 2. Pressure distribution of the liquid-solid interface $(z=0)$ at different times following the collision of a spherical water drop and a rigid plane. 


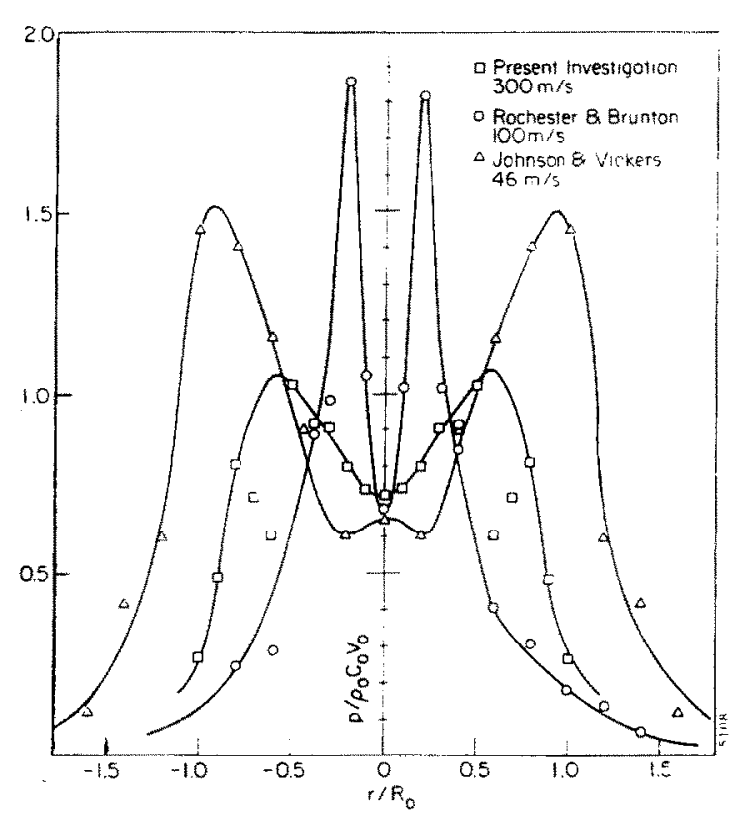

Fig. 3. Distribution of maximum pressure on the liquid-solid interface $(z=0)$. Data from the present investigation and published literature.

hammer pressure $[22,24]$. However, the difficulty of obtaining precise experimental information on this phenomenon is very considerable. Hence it must be admitted that the situation is not fully resolved at this time.

The time of application of the high pressure is extremely short. Figure 2 indicates non-dimensional times of less than unity. For water droplets of about $1 \mathrm{~mm}$ radius, the units of this non-dimensional time are of the order of microseconds. Hence the degree of damage to be expected from such very transient stressing of the material is problematical. Also the conventional mechanical material properties cannot be expected to be valid for such high rates of loading, and the effective failure stresses may be much greater than expected.

Figure 4 depicts the surface pressures resulting from conical droplet impact. In this case also the maximum contact pressure moves outward as the collision proceeds, finally reaching a value of about 1.4 times the waterhammer pressure. Again the duration of the entire process is about $1 \mu \mathrm{s}$ for a droplet radius of about $1 \mathrm{~mm}$.

Bulk and elastic modulus effects

General. The elastic modulus of the material impacted as well as the bulk modulus of the liquid affect the results as well as the geometrical effects previously discussed. This point leads to the general conclusion that the results from the mathematical models here discussed depend only upon:

(i) the Mach number of impact (referred to liquid sonic velocity, i.e. $\left.V / C_{0}\right)$; 


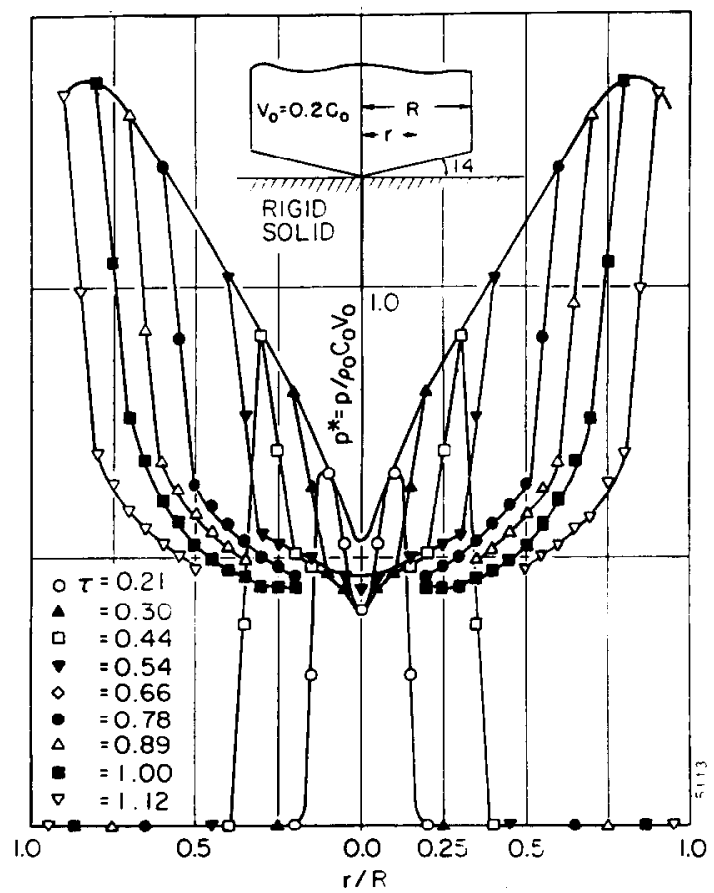

Fig. 4. Pressure distribution on the interface $(z=0)$ at different times following the collision of a conical water droplet and a rigid plane.

(ii) the rigidity of the impacted surface;

(iii) the geometry of the collision, i.e. the shape of the impacting aroplet and the impacted surface. The absolute droplet size is not required, except for the eventual computation of the absolute duration of effects. The resultant pressures are thus not a function of droplet size.

Bulk modulus of liquid. Any meaningful analysis of the liquid impact problem must obviously consider the liquid to possess a finite compressibility, i.e. an incompressible liquid model (infinite bulk modulus) would lead to a calculated infinite contact pressure for zero time since the liquid sonic velocity would then be infinite. Thus the water-hammer model requires a pressure wave speed $C$ in eqn. (1). For a relatively weak water hammer, it is adequate to assume $C \approx C_{0}$ where $C_{0}$ is the sonic speed in the liquid. However, this is not a good approximation for the impact case unless the impact liquid Mach number is very small, as is the case for a conventional water hammer. Equation (3) $[5,6,22]$ shows the pertinent correction to $C_{0}$ for water for impact velocities up to about $4500 \mathrm{~m} \mathrm{~s}^{-1}$. For an impact speed of $300 \mathrm{~m} \mathrm{~s}^{-1}$, i.e. liquid Mach number for water $\approx 0.2$, the shock wave speed is about 1.4 times the acoustic speed:

$$
C / C_{0}=1+2\left(V / C_{0}\right)-0.1\left(V / C_{0}\right)^{2}
$$


A correction to the density $\rho_{0}$ must also be considered, but this is relatively negligible except for very high impact Mach numbers.

Target material rigidity. The discussions above have all assumed a fully rigid flat material surface, although it is well known that finite target elasticity (or plasticity) will reduce the contact pressures somewhat, and hence will also reduce the stresses induced in the target material. As originally shown by DeHaller [25], the first order correction for material elasticity is provided by

$$
P=\frac{\rho_{0} C_{0} V}{1+\rho_{0} C_{0} / \rho_{\mathrm{s}} C_{\mathrm{s}}}
$$

where $\rho_{\mathrm{s}}$ and $C_{\mathrm{s}}$ are the density and acoustic speed of the solid material, and their product is the acoustic impedance. If the ratio of acoustic impedances between liquid and solid is appreciable, the reduction in impact pressure caused by the material elasticity effect can be large (Fig. 5).

The ratio of contact pressures between a collision of a liquid with an elastic solid and with a rigid solid [11] for the various cases computed in detail using a full mathematical model of the impact and assuming elastic response of the material are shown in Fig. 5. Spherical and cylindrical drops were considered with target materials of aluminum and Plexiglass* for a liquid Mach number of 0.2 . Figure 5 also shows the result from the classical DeHaller model (eqn. (4)), which appears to be conservative compared with the actual computer results, i.e. calculations using the DeHaller model will

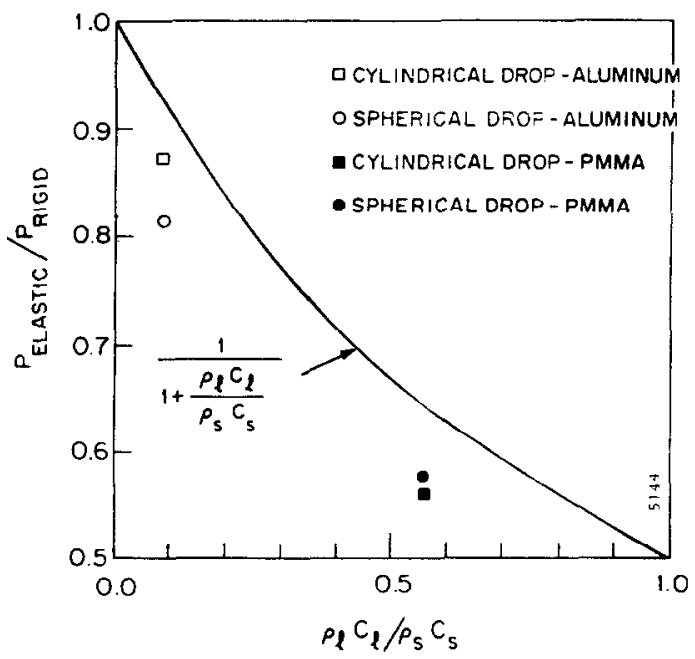

Fig. 5. The reduction of impact pressure as a function of the ratio of acoustic impedance.

*Polymethyl methacrylate (PMMA). 
overestimate the contact pressures to some extent. It is noted that the reduction in contact pressure for aluminum (as compared with a rigid solid) for these cases is about $15 \%$ and for Plexiglass it is about $45 \%$.

The computer calculations for Fig. 5 also provide the impact stresses and strains in the material as a function of position and time. These are reported in detail elsewhere $[8,9]$ and will not be repeated here. However, this type of calculation can be made for any material and liquid impact situation. From such results meaningful estimates of the probability and type of material failure to be expected can be made.

\section{Cavitation and impact erosion prediction techniques}

\section{General}

The previous section has discussed computer modelling of the basic phenomena and high speed photography as basic tools for developing the capability of predicting cavitation or liquid impact erosion rates. It may eventually become possible using this approach to estimate realistically the stress-time regimes existing in the material as a result of liquid impact or cavitation. This capability is closer for impact than for cavitation, since the cavitation phenomenon is basically considerably more complex. However, even if this capability should be attained, there would still remain the apparently even more difficult problem of accurately modelling the material removal process, given the pertinent time dependent stress-strain values. Computer modelling of the erosion process would obviously depend upon the type of material and the relative importance of corrosive effects as well as many other complicating factors, so that a general solution of this portion of the overall liquid erosion process seems even more remote than that of the fluid flow phenomena previously discussed. Nevertheless, continued, though relatively gradual, progress using computer modelling of the basic phenomena is certainly useful in increasing basic understanding of the phenomena involved and gradually improving overall predicting capabilities.

Since the capability for predicting erosion rates a priori from relatively basic principles does not yet exist, another possibility is to measure some easily and quickly measurable aspect of the cavitation or impact phenomenon and then to correlate this measurement with the measured damage. The measurement of noise provides such a possibility, which we believe to be of potential importance.

The use of noise for the detection of cavitation has been an accepted practice for many years, and more recently attempts have been made to correlate overall noise with erosion $[26,27]$. While some success has been obtained in specific experiments with individual pumps etc., it is our opinion that useful general correlations are not likely to be attained in this way. Hence we have here attempted to develop a technique whereby bubble collapse pressure pulse spectra are measured. If it is assumed that bubble collapse pressure pulse durations are roughly uniform (or at least uniquely 
related to the pressure magnitude), then the area under such a spectral curve provides a measure of the total impulse delivered to the damaged surface or to a probe presumably located at a position equivalent to that of the surface to be damaged from the viewpoint of the cavitation field. Such a technique is sensitive to not only the number of blows delivered to the surface but also to their strength. It thus appears to provide vital information beyond that provided either by a simple noise measurement or a noise frequency spectrum. Some small but successful experimentation with methods similar to those suggested here has been reported [28 - 30]. All work reported elsewhere [ 28 - 30] has used vibratory cavitation damage facilities rather than flowing systems. This is also true of our own work to date using this technique $[31,32]$.

Our experiments have included cavitation damage rate measurements in both water and molten sodium over a range of temperatures and suppression pressures in a $20 \mathrm{kHz}, 1.5 \mathrm{mil}(38 \mu \mathrm{m})$ vibratory horn cavitation damage facility. Detailed results are found elsewhere [31], but Fig. 6 illustrates the type of correlation obtained between measured bubble collapse pressure spectrum area and measured damage rate (MDPR is the mean depth of penetration, i.e. volume loss rate per unit exposed area). The best-fit relation is described by

$$
\operatorname{MDPR}=C(\text { spectrum area })^{n}
$$

where $C$ is a constant obtained empirically. The average value for the exponent $n$ is about 5 . The standard percentage error for these correlations is only about $20 \%$, which appears to us to be surprisingly small for experiments of this kind. We now plan to continue this type of experiment in a Venturi facility with water and also hopefully eventually in field devices, but no such experiments had been completed at the time of writing.

Although the evidence so far available is relatively sparse, it appears that an a priori erosion rate predicting technique, which can eventually be developed for application to many field devices, is virtually in hand. It would then be possible to predict from rapid and easy acoustic measurements eventual erosion rates which might be experienced in field devices. Such a capability, within even very rough limits of possible engineering utility, is unfortunately virtually non-existent today.

While the pressure pulse spectrum technique has so far [28 - 32] been applied only to cavitation erosion, it also appears applicable to impact erosion cases, depending of course on geometrical considerations. Its eventual application to rotating components also seems feasible, but obviously involves geometric considerations pertinent to specific applications.

\section{Conclusions} here.

The following overall conclusions can be drawn from the work reported 


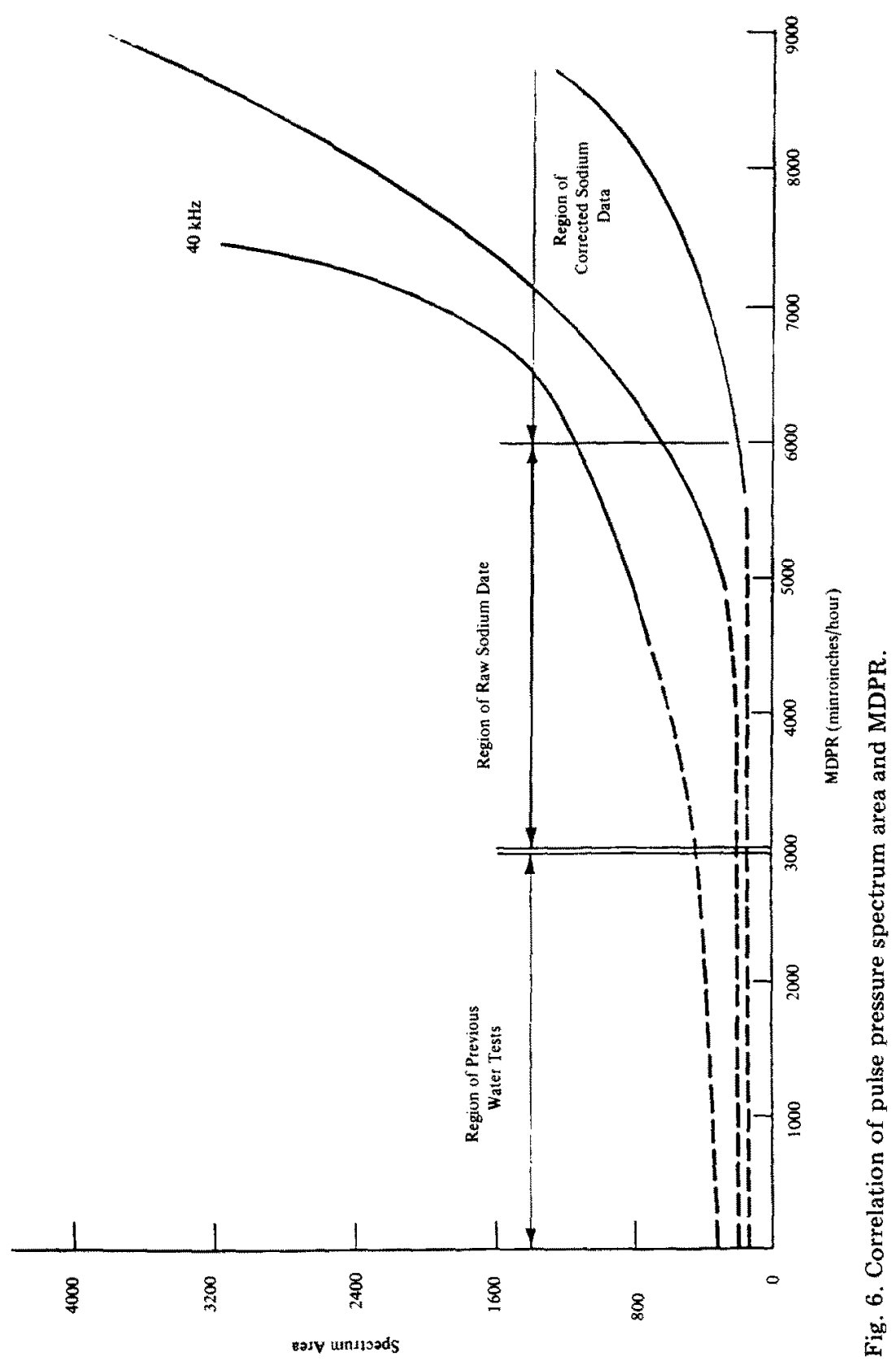


(1) Computer models of cavitation bubble collapse and also of liquid jet and droplet impact have been developed. These allow the computation in certain cases of the local stresses and strains as a function of time in the impacted material, but not of the eventual rates of erosion.

(2) The duration of material stresses and strains of damaging magnitude are only of the order of a few microseconds for cases of interest. Hence conventional mechanical material properties may not be pertinent.

(3) A technique for a priori prediction of eventual erosion rates based upon the measurement of individual pressure pulse spectra (number of blows versus magnitude) is under development. Initial results are extremely hopeful, apparently allowing the a priori prediction of such eventual erosion rates.

\section{Acknowledgments}

The work reported here was supported by many sources: National Science Foundation, Energy Research and Development Agency, Office of Naval Research, Alcoa Foundation and internal University of Michigan funds.

\section{References}

1 F. G. Hammitt, C. Chao, C. L. Kling and D. O. Rogers, ASTM round robin test with vibratory cavitation and liquid impact facilities of 6061-T-6511 aluminum alloy, 316 stainless steel, commercially pure nickel, ASTM Materials Research and Standards, ASTM, Philadelphia, Pa.

2 R. T. Knapp, J. W. Daily and F. G. Hammitt, Cavitation, McGraw-Hill, New York, 1970.

3 F. J. Heymann, Preliminary report on liquid impact erosion, round-robin test program, Internal Rep., Westinghouse Steam Turbine Division, July 1973.

4 F. G. Hammitt and F. J. Heymann, Liquid-erosion failures, in Failure Analysis and Prevention, Metals Handbook, 1975, Vol. 10, pp. 160 - 167.

5 Y. C. Huang, Numerical studies of unsteady, two-dimensional liquid impact phenomena, ORA Rep. No. UMICH 03371-8-T, June 1971.

6 Y. C. Huang, F. G. Hammitt and W. J. Yang, Hydrodynamic phenomena during highspeed collision between liquid droplet and rigid plane, J. Fluids Eng., 95 (1973) 276 294.

7 F. G. Hammitt and Y. C. Huang, Liquid droplet impingement studies at University of Michigan, Proc. Conf. Inst. Mech. Eng., London, pp. $237-243,1973$.

8 J. B. Hwang, The impact between a liquid drop and an elastic half-space, ORA Rep. No. UMICH 012449-5-T, March 1975.

9 J. B. Hwang and F. G. Hammitt, Transient distribution of the stress during the impact between a liquid drop and an aluminum body, Proc. 3rd Int. Symp. on Jet Cutting Technology, Chicago, May $11-13,1976$.

10 J. B. Hwang and F. G. Hammitt, High speed impact between curved liquid surface and rigid flat surface, ORA Rep. No. UMICH 012339-10-T, Oct. 1975.

11 J. B. Hwang, F. G. Hammitt and W. Kim, On liquid-solid impact phenomena, 1976 ASME Cavitation Forum, ASME, New York, 1976, pp. 24-27. 
12 R. D. Ivany, Collapse of a cavitation bubble in viscous, compressible liquid numerical and experimental analyses, ORA Rep. No. 03424-15-T, April 1965.

13 R. D. Ivany and F. G. Hammitt, Cavitation bubble collapse in viscous, compressible liquids -- numerical analyses, J. Basic Eng., (Dec. 1965) 977 - 985.

14 C. L. Kling, A high speed photographic study of cavitation bubble collapse, ORA Rep. No. UMICH 03371-2-T, March 1970;Ph.D. Thesis, University of Michigan, 197.

15 C. L. Kling and F. G. Hammitt, A photographic study of spark-induced cavitation bubble collapse, J. Basic Eng., 94D (4) (1972) 825 - 833.

16 E. E. Timm, An experimental photographic study of vapor bubble collapse and liquid jet impingement, ORA Rep. UMICH 01357-39-T 1974 ; Ph.D. Thesis, University of Michigan, 197.

17 T. M. Mitchell, Numerical studies of asymmetric and thermodynamic effects on cavitation bubble collapse, ORA Rep. No. UMICH 03371-5-T, Dec. 1970; Ph.D. Thesis, University of Michigan, 197.

18 T. M. Mitchell and F. G. Ilammitt, Asymmetric cavitation bubble collapse, J. Fluids Eng., 95 (1) (1973) 29 - 37.

19 T. M. Mitchell and F. G. Hammitt, On the effects of heat transfer upon collapsing bubbles, Nucl. Sci. Eng., 53 (3) (1974) $263-276$.

20 C. G. Naude and A. T. Ellis, On the mechanism of cavitation damage by non-hemispherical cavities collapsing in contact with a solid boundary, J. Basic Eng., 83D (1961) 648 - 656 .

21 N. D. Shutler and R. B. Mesler, A photographic study of the dynamics and damage capabilities of bubbles collapsing near solid boundaries, J. Basic Eng., 87D (1965) $511-517$.

22 W. Johnson and G. W. Vickers, Transient stress distribution caused by water-jet impact, J. Mech. Eng. Sci., 15 (4) (1973) 302 - 310.

23 F. J. Heymann, On the shock wave velocity and impact pressure in high-speed liquid solid impact, J. Basic Eng., 90D (1968) $400-402$.

24 M. C. Rochester and J. H. Brunton, Surface pressure distribution during drop impingement, Proc. 4th Int. Conf. on Rain Erosion and Associated Phenomena, Meersburg, Germany, May 1974.

25 P. DeHaller, Untersuchungen über die durch Kavitation hervorgerufenen Korrosionen, Schweiz. Bauztg., 101 (1933) 243.

26 I. S. Pearsall and P. J. McNulty, Comparisons of cavitation noise with erosion, 1968 ASME Cavitation Forum, ASME, New York, 1968, pp. 6 - 7.

27 J. J. Varga and Gy. Sebestyen, Determination of hydrodynamic cavitation intensity by noise measurement, Proc. 2nd Int. JSME Symp. on Fluid Machinery and Fluidics, Tokyo, Sept. 1972, Jpn. Soc. Mech. Eng., Tokyo, 1972, pp. $285-292$; see also J. J. Varga, Gy. Sebestyen and A. Fay, Detection of cavitation by acoustic and vibrationmeasurement methods, Houille Blanche, 2 (1969) 137 - 149.

28 F. Numachi, Transitional phenomena in ultrasonic shock waves emitted by cavitation on hydrofoils, J. Basic Eng., (June 1959) 153.

29 F. Numachi, An experimental study of accelerated cavitation induced by ultrasonics, J. Basic Eng., 87 (1965) 967 - 976.

30 V. K. Muckarov, A. A. Kortnev, S. G. Suprun and G. I. Okolelov, Cavitation erosion spectra analysis of pulse-heights produced by cavitation bubbles, Proc. 6 th NonLinear Acoustics Conf., Moscow, July 1975, Odessa Poly technic Institute, 1975.

31 F. G. Hammitt, J. B. Hwang, M. K. De, A. N. El Hasrouni, S. A. Barber and G. V. Chammas, Final report - Argonne National Laboratory, ORA Rep. No. UMICH $013503-2-F$, June 1976.

32 F. G. Hammitt and J. B. Hwang, Ultrasonic cavitation regime pulse-count spectra as related to cavitation erosion, Proc. 7 th Int. Non-Linear Acoustics Conf., Blacksburg, Va., Aug. 1976. 